NASA Technical Memorandum 102431

ICOMP-89-30

\title{
Spatial Evolution of Nonlinear Acoustic Mode Instabilities on Hypersonic Boundary Layers
}

M.E. Goldstein

Lewis Research Center

Cleveland, Ohio

and

D.W. Wundrow

State University of New York at Buffalo

Buffalo, New York

December 1989

\section{N/Sก}

(NASA-TM-102431) SPATIAL EVJLUTION OF NINLINEAR ACCUSTIC MONE INSTADILITIES ON HYPFRSUNIC DUUNOAOY LAYERS (NASA) $30 \mathrm{p}$ CSCL 200

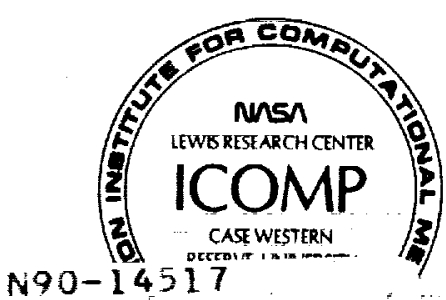


SPATIAL EVOLUTION OF NONLINEAR ACOUSTIC MODE INSTABILITIES

ON HYPERSONIC BOUNDARY LAYERS*

\author{
M.E. Goldstein \\ National Aeronautics and Space Administration \\ Lewis Research Center \\ Cleveland, Ohio 44135 \\ and \\ D.W. Wundrow** \\ State University of New York at Buffalo \\ Buffalo, New York 14222
}

곤

\begin{abstract}
We consider the effects of strong critical layer nonlinearity on the spatial evolution of an inftially linear "acoustic mode" instability wave on a hypersonic flat plate boundary layer. Our analysis shows that nonlinearity, which is initially confined to a thin critical layer, first becomes important when the amplitude of the pressure fluctuations become $O\left(1 / M^{4} \ln M^{2}\right)$, where $M$ is the free stream Mach number. The flow outside the critical layer is still determined by linear dynamics and therefore takes the form of a linear instability wave - but with its amplitude completely determined by the flow within the critical layer. The latter flow is determined by a coupled set of nonlinear equations, which we had to solve numerically.

1. INTRODUCTION

Laminar boundary layer instabilities are predominantly inviscid at sufficiently high Mach numbers (Mack, 1984, 1987) with the so-called vorticity modes exhibiting the most rapld growth at very large Mach numbers. The
\end{abstract}

\footnotetext{
*Work partially funded by NASA Grant NGT-50085, Space Act Agreement C99066G, and NASA Contract NAS3-25266.

**Presently employed by Sverdrup Technology, Inc., NASA Lewis Research Center Group, Cleveland, Ohio 44135.
} 
so-called acoustic modes exhibit the most rapid growth at more moderate Mach numbers - with the two-dimensional disturbances growing more rapidly than the corresponding oblique waves.

Mack (1984, 1987) computed the relevant numerical solutions to Rayleigh's equation and Cowley and Hall (1988) worked out the corresponding asymptotic solution for the hypersonic limit where the free stream Mach number $M \rightarrow \infty$. Their results, as well as those of Mack (1984, 1987), suggest that, while the instability wave number becomes small, the instability wave growth rate becomes even smaller as $M \rightarrow \infty$ which means that there is a well-defined critical layer at every unstable frequency in this limit. Nonlinear effects will then balance the resulting singularity at sufficiently large Reynolds numbers and the present work is concerned with extending the Cowley-Hall (1988) analysis into this nonlinear regime.

Boundary-layer-transition experiments often involve spatially growing instability waves generated by relatively two-dimensional, single-frequency excitation devices such as vibrating ribbons or acoustic speakers. As the instability wave propagates downstream, its amplitude continues to increase until nonlinear effects come into play - provided, of course, that the initial amplitude is sufficiently large and/or the mean flow divergence is sufficientiy small (i.e., the Reynolds number is sufficiently large).

Our previous remarks suggest that the nonlinearity first comes into play locally, i.e., within a critical layer. The solution to the resulting nonlinear critical layer problem reduces to the linear small growth rate hypersonic instability wave solution far upstream, and is therefore the natural downstream continuation of this upstream linear solution. The flow outside the critical layer continues to be linear but the corresponding instablitity wave amplitude is completely determined by the nonlinear dynamics with the critical 
layer. These considerations fix the relative scaling between instability wave amplitude and Mach number and thereby show that the nonlinearlty first becomes important when the pressure fluctuation amplitude becomes $O\left(1 / M^{4} \ln M^{2}\right)$ in the main boundary layer.

The critical layer nonlinearity is strong in the sense that it enters through a coefficient in the lowest order equation (as in the Goldstein, Durbin, and Leib, 1987 incompressible boundary layer analysis) rather than through an inhomogeneous higher order term (as in the Goldstein and Leib, 1989 compressible shear layer analysis). However, it differs from the former analysis (but is similar to the latter) in that coupled vorticity and energy equations have to be solved simultaneously. This has been done numerically. While nonlinear effects always decrease the instability wave growth in the Goldstein et al. (1987) analysis, compressibility effects produce a dramatic increase in growth in the present analysis - leading to a possible singularity at a finite downstream position (as found by Goldstein and Leib (1989)). Finally it is worth noting that there is an unusual nonlinear modulation of the basic critical layer streamline pattern which does not usually occur in nonlinear critical layer analyses.

The overall plan of the paper is as follows. The problem is formulated in section 2, where we show how the nonlinear flow gradually evolves from the strictly linear hypersonic solution. The flow outside the critical layer is a linear inviscid perturbation about a hypersonic ( $1 . e ., M \gg 1)$ boundary layer flow, and is found by extending the asymptotic analysis of Cowley and Hall (1988) into the nonlinear regime. This flow has a double layer structure and the solution for the main boundary layer region is worked out in section 3 . The critical layer is contained in the outer region and in section 4 we obtain the outer region solution that applies outside the critical layer. This is 
then used to formulate the relevant critical layer problem in section 5 . The resulting critical layer vorticity and energy equations are different from the usual nonlinear nonequilibrium transport equations but can be transformed into the latter by a suitable change of independent variables. The numerical solution of these coupled equations is described in section 6. Finally, numerical results are discussed in section 7.

\section{FORMULATION}

The free stream flow parameters are used as reference quantities and are generally denoted by the subscript $\infty$. The reference length, say $\Delta$ is taken to be some suitable boundary layer thickness (e.g., momentum thickness). Then the steady flow is characterized by the Mach number

$$
M \equiv U_{\infty} / C_{\infty}
$$

and Reynolds number

$$
\operatorname{Re} \equiv U_{\infty} \Delta / U_{\infty} \text {, }
$$

where

$$
\left.C_{\infty}=\left(\gamma R T_{\infty}\right)\right)^{1 / 2}
$$

is the speed of sound in the free stream, $v$ is the kinematic viscosity, $Y$ is the isentropic exponent of the gas, and $R$ is the gas constant.

We suppose that the flow is two dimensional and that $R e$ is large enough that the unsteady motion is essentially inviscid and unaffected by mean boundary layer growth over the region in which nonlinear interaction takes place. We can then suppose that the mean pressure is constant and that the mean flow velocity $U(y)$ depends only on the transverse coordinate $y$ to the required order of approximation. We assume that the wall is insulated and, for simplicity, restrict our attention to an ideal gas which satisfies Southerland's viscosity law and has Prandtl number unity. In this case the local mean density $R$ and mean temperature $T$ will be related by 


$$
R T=1,
$$

and the mean velocity and temperature will be given by

$$
U=h^{\prime}(n)
$$

and

$$
T=1+\frac{\gamma-1}{2} M^{2}\left(1-U^{2}\right)
$$

respectively, where $n$ is the Dorodnitsy-Howarth variable (Stewartson, 1964) defined by

$$
\eta \equiv \int_{0}^{y} \frac{d y}{T}
$$

the prime denotes differentiation with respect to $n$, and $h$ is the Blasius function, i.e., it satisfies

$$
h h^{\prime \prime}+2 h^{\prime \prime \prime}=0 .
$$

It follows that (Schilichting, 1960, pp. 117 and 118)

$$
U=1-\frac{b}{\tilde{n}} e^{-\tilde{n}^{2} / 4}\left[1-\frac{2}{\tilde{n}^{2}}+0\left(\tilde{n}^{-4}\right)\right]-\frac{b^{2} e^{-\tilde{n}^{2} / 2}}{\tilde{n}^{4}}+\cdots,
$$

as $\tilde{\eta} \rightarrow \infty$, where

$$
\tilde{n} \equiv \eta-\beta
$$

$\beta \simeq 1.73$, and $b \simeq 0.462$.

As indicated in section 1 , we suppose that the unsteady motion starts as a linear inviscid instability wave (which is governed by Rayleigh's equation) in the upstream region where $x \rightarrow-\infty$. We also suppose that the linear mode is of the acoustlc type (Mack, 1984, 1987) and that

$$
\sigma \equiv \frac{1}{M^{2}}<1
$$


The relevant asymptotic solution to Rayleigh's equation was worked out to lowest approximation by Cowley and Hall (1988). They show that the scaled complex wave number $\alpha$ is $O(\sigma)$, that the phase speed $c$ behaves like

$$
c=1-\sigma \bar{c} \sim 1 \quad \text { ds } \quad \sigma \rightarrow 0,
$$

where $\bar{c}=0(1)$, and that the flow develops a double (actually triple if the free stream is included) layered structure, with a relatively thin outer region, where

$$
\ln \tilde{Y} \equiv \delta(2 \delta-\tilde{\eta})
$$

is order unity provided the constant $\delta$ satisfies

$$
\frac{e^{-\delta^{2}}}{2 \delta}=\sigma
$$

Cowley and Hall (1989) extended their analysis to higher order in $\sigma$ to show that the instability wave growth rate cor imaginary part of the complex wave number) is small relative to $\operatorname{Re} \alpha$, or more precisely that

$$
\ln \alpha=0\left(\frac{\sigma^{3}}{\delta}\right) \text {. }
$$

The linear instability wave will then have a distinct critical layer. which, in view of (2.9), (2.12), and (2.13), must lie in the outer region where $\tilde{Y}=0(1)$, and

$U=1-a b \tilde{Y}\left[1-\frac{1}{2 \delta^{2}}\left(1-\ln \tilde{Y}+\frac{1}{2} \ln ^{2} \tilde{Y}\right)+o\left(\delta^{-3}\right)\right]-\left(\frac{a b \tilde{Y}}{2 \delta}\right)^{2}+\cdots \cdot$

We expect the analysis to become nonlinear in this layer because the flow is assumed to be inviscid and the linear instability wave amplitude increases in the downstream direction. The motion is predominantly linear outside the critical layer and we expect the solution there to expand like

$$
u=U(n)+\varepsilon u_{1}+\varepsilon^{2} u_{2}+\ldots
$$




$$
\begin{gathered}
v=\varepsilon v_{1}+\varepsilon^{2} v_{2}+\ldots \\
\theta=T(n)+\varepsilon \tau_{1}+\varepsilon^{2} \tau_{2}+\ldots . \\
p^{1 / \gamma}=1+\varepsilon \pi_{1}+\varepsilon^{2} \pi_{2}+\ldots .
\end{gathered}
$$

where $\{u, v\}$ are the velocity components in the $x$ and $y$ directions, $\theta$ is the temperature, $p$ is the pressure, and $\varepsilon$ is a characteristic amplitude of the instability wave in the streamwise region where nonlinearity first becomes important. It will be specified more precisely below. Then since $\left\{u_{1}, v_{1}, \tau_{1}, p_{1}\right\}$ are determined by 1 inear dynamics, it follows from Goldstein (1984) that the expansion coefficients in (2.17) to (2.20) (which depend on $x$, $\eta, t$, and $\sigma$ ) are determined by

$$
\begin{gathered}
L \pi_{1}=0 \\
L \pi_{2}=\frac{D}{D t}\left(\frac{\partial f}{\partial x}+\frac{\partial g}{\partial y}\right)-\frac{2 U^{\prime}}{T} \frac{\partial g}{\partial x} \\
\frac{D}{D t} u_{1}+\frac{U^{\prime}}{T} v_{1}=-\sigma T \frac{\partial \pi_{1}}{\partial x} \\
\frac{D}{D t}\left(u_{2}+u_{1} \pi_{1}\right)+\frac{U^{\prime}}{T}\left(v_{2}+v_{1} \pi_{1}\right)=-\sigma T \frac{\partial \pi_{2}}{\partial x}-f \\
\frac{D v_{1}}{D t}=-\sigma T \frac{\partial \pi_{2}}{\partial y} \\
\frac{D}{D t}\left(v_{2}+v_{1} \pi_{1}\right)=-\sigma T \frac{\partial \pi_{2}}{\partial y}-g \\
\frac{D}{D t} \tau_{1}+T^{\prime} v_{1}=(\gamma-1) T \frac{D \pi_{1}}{D t}
\end{gathered}
$$

and a similar equation for $\tau_{2}$, where the prime still denotes differentiation with respect to $n$,

$$
L \equiv \frac{D}{D t}\left(\frac{D^{2}}{D t^{2}}-\sigma \nabla \cdot T \nabla\right)+2 \sigma U^{\prime} \frac{\partial^{2}}{\partial x \partial y},
$$




$$
\begin{gathered}
\frac{D}{D t} \equiv \frac{\partial}{\partial t}+U \frac{\partial}{\partial x}, \\
\nabla \equiv\left\{\frac{\partial}{\partial x}, \frac{\partial}{\partial y}\right\}, \\
f \equiv \frac{\partial}{\partial x} u_{1}^{2}+\frac{\partial}{\partial y} u_{1} v_{1}+\sigma \tau_{1} \frac{\partial}{\partial x} \pi_{1},
\end{gathered}
$$

and

$$
g \equiv \frac{\partial}{\partial x} u_{1} v_{1}+\frac{\partial}{\partial y} v_{1}^{2}+\sigma \tau_{1} \frac{\partial}{\partial y} \pi_{1}
$$

It now follows from (2.12), (2.15), and the fact that $\alpha=0(\sigma)$ that the solution to $(2.21),(2.23),(2.25)$, and (2.27) that matches onto the upstream linear solution must be of the form

$$
\begin{gathered}
u_{1}=\frac{\delta}{\sigma} \operatorname{Re} \Psi_{1}\left(n, x_{1}\right) A^{\dagger}\left(x_{1}\right) e^{i \bar{x}}, \\
v_{1}=-\bar{\alpha} \operatorname{Re} i \Phi_{1}\left(n, x_{1}\right) A^{\dagger} e^{i \bar{x}}, \\
\tau_{1}=\frac{\delta}{\sigma^{2}} \operatorname{Re} \Theta_{1} A^{\dagger} e^{i \bar{x}}, \\
\pi_{1}=\operatorname{Re} \Pi_{1} A^{\dagger} e^{i \bar{x}}
\end{gathered}
$$

where we have put

$$
\begin{gathered}
x_{1}=\frac{\sigma^{3} x}{\delta}, \\
\bar{x}=\sigma \bar{\alpha}[x-(1-\sigma \bar{c}) t],
\end{gathered}
$$

$A^{\dagger}\left(x_{1}\right), \bar{\alpha}(\sigma)$, and $\bar{c}(\sigma)$ are real quantities and, to the required level of approximation, $\Pi_{1}$ satisfies Rayleigh's equation

$$
\mathscr{L}_{1} \Pi_{1}=0
$$

where 


$$
\mathscr{L}_{n} \equiv(U-c)^{2} \frac{d}{d n} \frac{1}{(U-c)^{2}} \frac{d}{d n}-(n \alpha)^{2} T\left[T-\frac{1}{\sigma}(U-c)^{2}\right] \quad \text { for } n=1,2, \ldots
$$

are the linear Rayleigh operators, the complex wave number and phase speed $\alpha$ and $c$, respectively, are given by

$$
\begin{gathered}
\alpha=\sigma \bar{\alpha}+\frac{\sigma^{3}}{\delta} \frac{A^{\dagger^{\prime}}}{i A^{\dagger}}, \\
c=1-\sigma \bar{c}-\frac{\sigma^{2}}{\delta \bar{\alpha}} \frac{A^{\dagger^{\prime}}}{i A^{\dagger}},
\end{gathered}
$$

and the prime now denotes differentiation with respect to $x_{1}$. We note in passing that $\bar{\alpha}$ has an expansion of the form

$$
\bar{\alpha}=\bar{\alpha}_{1}+\sigma \bar{\alpha}_{2}+\sigma^{2} \bar{\alpha}_{3}+\ldots .
$$

where each of the coefficients has its own series expansion* in $\delta^{-1}, \ln \delta$, etc. (see (2.14)) and similarly for $\bar{c}, i . e$. ,

$$
\begin{gathered}
\bar{\alpha}_{n}=\bar{\alpha}_{n}^{(0)}+\frac{1}{\delta} \bar{\alpha}_{n}^{(1)}+\ldots . \\
\bar{c}_{n}=\bar{c}_{n}^{(0)}+\frac{1}{\delta} \bar{c}_{n}^{(1)}+\ldots .
\end{gathered}
$$

$\Pi_{1}$ must satisfy the boundary conditions

$$
\frac{d \Pi 1}{d n}=0 \quad \text { at } \quad n=0 \text {, }
$$

and

$$
\Pi_{1} \rightarrow 0 \text { as } n \rightarrow \infty
$$

and, in order to match with the linear solution far upstream, we must require that

*It might be helpful to think of $\sigma$ and $\delta$ as independent expansion coefficients at this point. 


$$
A^{\dagger} \rightarrow e^{k x_{1}} \quad \text { as } \quad x_{1} \rightarrow-\infty
$$

where $k$ is the scaled growth rate of the upstream linear instability wave and a is a complex constant. Finally, the remaining functions of $\eta$, i.e., $\Psi_{1}, \Phi_{1}$, and $\Theta_{1}$ can readily be found from $(2.23),(2.25)$, and (2.27) once $\Pi_{1}$ is known.

In the following two sections we derive dispersion relations for the instability wave amplitude outside the critical layer.

\section{LINEAR SOLUTION IN MAIN BOUNDARY LAYER}

First suppose that $\eta=O(1)$. It is easy to see from the Cowley-Hall (1988) analysis that $\Pi_{1}$ should expand like

$$
\Pi_{1}=\frac{1}{\sigma}\left(P_{0}+\sigma P_{1}+\sigma^{2} P_{2}+. . \cdot\right) \text {, }
$$

where each of the expansion coefficients in turn has the expansion

$$
p_{n}=p_{n}^{(0)}+\frac{1}{\delta} p_{n}^{(1)}+\frac{1}{\delta^{2}} p_{n}^{(2)}+\ldots \text { for } n=1,2, \ldots . .
$$

Substituting these into (2.39) and equating coefficients of like powers of $\sigma$ and $\delta$, we find that

$$
\begin{aligned}
& \mathscr{S}_{1}^{(0)} \mathrm{P}_{0}=0, \\
& \mathscr{L}_{1}^{(0)} P_{1}^{(0)}=Q_{1} \frac{(1-U)^{2}}{P_{0}} \equiv \bar{\alpha}_{1}(y-1)\left(1-U^{2}\right)\left\{\left[\frac{y-1}{2}\left(1-U^{2}\right)-(U-1)^{2}\right] \bar{\alpha}_{2}\right. \\
& \left.+\left[1-\frac{1}{\gamma-1}\left(\frac{1-U}{1+U}\right)+\bar{c}_{1}(1-U)\right] \bar{\alpha}_{1}\right\} P_{0}-\frac{2 \bar{c}_{1} U^{\prime}}{(1-U)^{2}} \frac{d P_{0}}{d \eta} \text {. }
\end{aligned}
$$

and

$$
\mathscr{L}_{1}^{(0)} P_{2}^{(1)}=Q_{2} \frac{(1-U)^{2}}{P_{0}}
$$

where 


$$
Q_{2} \equiv Q_{2 r}-1 q \frac{A^{\dagger^{\prime}}}{A^{\dagger}}
$$

$Q_{2 r}, q$ are real,

$$
q \equiv(Y-1)(1+U) \bar{\alpha}_{1}\left[\frac{Y-1}{2}(1+U)+U\right] P_{0}^{2}+\frac{U^{\prime}}{\bar{\alpha}_{1}(1-U)^{4}} \frac{d P_{0}^{2}}{d \eta} .
$$

and we have put

$$
\begin{aligned}
\mathscr{L}_{1}^{(0)} \equiv(1-U)^{2} \frac{d}{d n} & \frac{1}{(U-1)^{2}} \frac{d}{d \eta} \\
& -\alpha_{1}^{2}\left(\frac{y-1}{2}\right)\left(1-U^{2}\right)\left[\frac{Y-1}{2}\left(1-U^{2}\right)-(U-1)^{2}\right] .
\end{aligned}
$$

Equation (3.3) was solved numerically by Cowley and Hall (1988). They point out that

$$
P_{0} \sim \frac{D}{\tilde{n}^{3}} e^{-\tilde{n}^{2} / 2} \quad \text { as } \quad \eta \rightarrow \infty
$$

where $D$ is an, as yet, undetermined constant. Equations (3.4) and (3.5) can now be solved by variation of parameters to obtain

$$
\begin{aligned}
& P_{1}=P_{0} \int\left(\frac{1-U}{P_{0}}\right)^{2} \int_{0}^{n} Q_{1}(\hat{n}) d \hat{n} d n, \\
& P_{2}^{(1)}=P_{0} \int\left(\frac{1-U}{P_{0}}\right)^{2} \int_{0}^{n} Q_{2}(\hat{n}) \hat{n} d n .
\end{aligned}
$$

It follows that

$$
P_{1}^{(0)} \sim \frac{b^{2}}{D} \int_{0}^{\infty} Q_{1} d n ; P_{2}^{(1)} \sim \frac{b^{2}}{D} \int_{0}^{\infty} Q_{2} d n, \quad \text { as } \quad n \rightarrow \infty . \quad(3.11-a, b)
$$




\section{LINEAR SOLUTION IN THE EDGE LAYER}

Cowley and $\mathrm{Hall}$ (1988) point out that the expansion (3.1) breaks down at large disturbances from the wall and then proceed to construct a new "outer" solution for the region where $\tilde{Y}=0(1)$ (see $(2.13)$ ). The expansion in this region must be of the form

$$
\Pi_{1}=1+\left(\frac{\sigma}{\delta}\right) \tilde{\mathrm{p}}_{1}+\left(\frac{\sigma}{\delta}\right)^{2} \tilde{\mathrm{P}}_{2}+\ldots .
$$

where again each expansion coefficient has its own series expansion

$$
\tilde{P}_{n}=\delta \tilde{P}_{n}^{(-1)}+\tilde{P}_{n}^{(0)}+\frac{1}{\delta} \tilde{P}_{n}^{(1)}+\ldots .
$$

in terms of $\delta^{-1}$, in $\delta$, etc. Substituting this, together with the new varlable (2.13), into (2.39) and equating coefficients of $(\sigma / \delta)$, we find upon integration that

$$
\begin{aligned}
& \tilde{p}_{1}^{(-1)}=\tilde{E}_{1}^{(-1)} \\
& \tilde{P}_{1}^{(0)}=\tilde{B}_{1}^{(0)}\left(\frac{b^{2} \tilde{Y}^{2}}{2}-2 c_{1} b \tilde{Y}+c_{1}^{2} \ln \tilde{Y}\right)+\tilde{E}_{1}^{(0)} \text {. } \\
& \tilde{p}_{2}^{(0)}=\left[\frac{\bar{\alpha}_{1}^{2}}{\bar{c}_{1}} T_{c}^{2}+2\left(\bar{c}_{2}^{(1)}+\frac{1}{\bar{\alpha}_{1}} \frac{A^{\dagger^{\prime}}}{i^{\dagger}}\right) \tilde{B}_{1}^{(0)}\right]\left(\bar{c}_{1} \ln \tilde{Y}-b \tilde{Y}\right) \\
& +\frac{\bar{\alpha}_{1}^{2}}{\bar{c}_{1}^{2}}\left\{b \int_{0}^{Y}\left(b \tilde{Y}-2 \bar{c}_{1}\right) \ln \tilde{Y} d \tilde{Y}+\frac{\bar{c}_{1}^{2}}{2} \ln ^{2} \tilde{Y}-\left[1-\bar{c}_{1}^{2}(Y-1)^{2}\right]\right. \\
& \left.x \int \frac{\left(b \tilde{Y}-c_{1}\right)^{2}}{\tilde{Y}}\left[\phi^{ \pm}+\ln \left|b \tilde{Y}-\bar{c}_{1}\right|\right] d \tilde{Y}\right\}+\tilde{E}_{2},
\end{aligned}
$$

where we note that the lowest order solution (i.e., $\tilde{p}_{1}$ ) is given in cowley and Hall (1988), $\tilde{B}_{1}, \tilde{E}_{1}, \tilde{E}_{2}$, and $\phi^{ \pm}$are constants of integration (the latter of which can be different depending on whether $\tilde{Y} \gtrless 0$ ), and 


$$
T_{c} \equiv 1+(\gamma-1) \bar{c}_{1}
$$

is the mean temperature at the critical level where

$$
b \tilde{Y}=\bar{c}_{1} .
$$

This solution does not satisfy appropriate free stream boundary conditions and it is necessary to introduce another outer expansion for the region where the variable

$$
\hat{n} \equiv \text { on }
$$

is order one. The solution in this region is

$$
\Pi_{1}=\left(1+\sigma \hat{B}_{1}\right) e^{-\bar{\alpha} \sqrt{1-\sigma \bar{C}_{1}^{2}} \hat{n}}+\ldots
$$

to the required order of accuracy and matching with (4.1) shows that

$$
\tilde{B}_{1}^{(0)}=\frac{\bar{\alpha}_{1}}{\bar{c}_{1}^{2}}
$$

and

$\tilde{E}_{1}^{(0)}=\bar{\alpha}_{1}\left\{T_{c}^{2}-\left[1-\bar{c}_{1}^{2}(\gamma-1)^{2}\right]\left[\phi^{-}+\ln \left|c_{1}\right|\right]\right\}+\frac{2}{\bar{c}_{1}}\left(\bar{c}_{2}^{(1)}+\frac{1}{\bar{\alpha}_{1}} \frac{A^{\dagger^{\prime}}}{i A^{\dagger}}\right)$.

Finally, matching (3.1) and (4.1) and using (3.9) and (3.11) shows that

$$
\begin{gathered}
D=\frac{b^{2} \bar{\alpha}_{1}}{\bar{c}_{1}^{2},} \\
1=\frac{\bar{c}_{1}^{2}}{\bar{\alpha}_{1}} \int_{0}^{\infty} Q_{1} d n, \\
\eta_{m} \phi^{+}=0,
\end{gathered}
$$

and 


$$
\bar{\alpha}_{1}^{2} \bar{c}_{1}\left[1-\bar{c}_{1}^{2}(y-1)^{2}\right] 2 m \phi^{-}+2 \frac{A^{\dagger^{\prime}}}{A^{\dagger}}=\bar{c}_{1}^{3} \frac{A^{\dagger^{\prime}}}{A^{\dagger}} \int_{0}^{\infty} q d \eta .
$$

Substituting (2.13), (2.34), (2.36), (2.41), (2.42), (4.3), and (4.4) into (2.25) and equating coefficients of like powers of $\sigma$, we find

$$
\Phi_{1}=\frac{\bar{\alpha}_{1}(\bar{c}-b \tilde{Y})}{\left(\bar{\alpha} \bar{c}_{1}\right)^{2}}+\left(\frac{\sigma}{\delta}\right) \frac{1}{\bar{c}_{1}}\left(T_{c}^{2}+\frac{1}{\bar{\alpha}_{1}^{2} \bar{c}_{1}} \frac{A^{\dagger^{\prime}}}{A^{\dagger}}\right)+\ldots .
$$

Substituting this together with (2.33) and the previous equations into (2.23) and using (2.9)

$$
\begin{aligned}
& \Psi_{1}=\frac{\bar{\alpha}_{1}}{T\left(\bar{\alpha} \bar{c}_{1}\right)^{2}}(b \tilde{Y}+\ldots)+\frac{\sigma}{\delta}\left\{\frac{T}{\left(\bar{c}_{1}-b \tilde{Y}\right)}\left[\frac{b \tilde{Y}}{c_{1}}\left(\frac{T c}{T}\right)^{2}-1\right]+\frac{b \tilde{Y}}{T-2}[\ln \tilde{Y}\right. \\
& \left.\left.-\left[1-\bar{c}_{1}^{2}(y-1)^{2}\right]\left(\phi^{ \pm}+\ln \left|b \tilde{Y}-\vec{c}_{1}\right|\right)\right]\right\}+\ldots .
\end{aligned}
$$

And finally, proceeding similarly with (2.35) and (2.27), we obtain

$$
\Theta_{1}=-\frac{\bar{\alpha}_{1}(\gamma-1)}{T\left(\bar{\alpha} \bar{c}_{1}\right)^{2}} b \tilde{Y}+. . .
$$

\section{NONLINEAR EDGE LAYER TERMS}

The lowest approximation (in terms of $\sigma$ ) of the $O\left(\varepsilon^{2}\right)$ terms in the expansions (2.17) to (2.20) has to be determined before the solution within the critical layer can be found. However, it is only necessary to consider the edge layer solution for this purpose. It follows from (2.33) to (2.36) that this solution must be of the form

$$
\pi_{2}=\Pi_{2}^{(0)}+\operatorname{Re} \Pi_{2}^{(2)} e^{2 i \bar{X}}
$$




$$
v_{2}+v_{1} \pi_{1}=-2 \bar{\alpha}\left[\Phi_{2}^{(0)}+\operatorname{Re} i_{2}^{(2)} e^{2 i \bar{x}}\right],
$$

and

$$
u_{2}+u_{1} \pi_{1}=\frac{\delta}{\sigma}\left[\Psi_{2}^{(0)}+\operatorname{Re} \Psi_{2}^{(2)} e^{2 i \bar{x}}\right]
$$

where the, as yet, unknown coefficients are functions of $n, x_{1}$, and $\sigma$. Substituting (5.1) into (2.22) and using (2.31), (2.28) to (2.36), and (2.40), we find

$$
\mathscr{L}_{2} \Pi_{2}^{(2)}=-\frac{1}{\sigma}\left\{2 i \alpha T F+(U-c)^{2} \frac{d}{d \eta}\left[\frac{G}{(U-c)^{2}}\right]\right\} \text {. }
$$

where

$$
F \equiv \frac{i \alpha}{2}\left(\frac{\delta}{\sigma}\right)^{2}\left[2 \Psi_{1}^{2}-\frac{1}{\mathrm{~T} \delta} \frac{d}{d \eta}\left(\Phi_{1} F_{1}\right)+\frac{\sigma}{\delta} \Theta_{1} \Pi_{1}\right] A^{\dagger 2},
$$

and

$$
G \equiv \frac{1}{2} \frac{\delta}{\sigma^{2}}\left(2 \alpha^{2} \Phi_{1} \Psi_{1}-\frac{\alpha^{2}}{\delta T} \frac{d}{d \eta} \Phi_{1}^{2}+\sigma \frac{\Theta_{1}}{T} \frac{d \Pi_{1}}{d \eta}\right) A^{\dagger 2} .
$$

Substituting (2.6), (2.13), (2.16), (2.40) to (2.43), and (4.1), (4.3), and (4.15) to (4.17) into this result we find that

$\tilde{Y}\left(\bar{c}_{1}-b \tilde{Y}\right)^{2} \frac{d}{d \tilde{Y}} \frac{\tilde{Y}}{\left(\bar{c}_{1}-b \tilde{Y}\right)^{2}} \frac{d \Pi_{2}^{(2)}}{d \tilde{Y}}=\frac{1}{\sigma \bar{c}_{1}^{4}}\left[\frac{b \tilde{Y}}{T}\left(b \tilde{Y}+\frac{\bar{c}_{1}-b \tilde{Y}}{\bar{T}}\right)\right.$

$$
\left.-\frac{b \tilde{Y}(Y-1)\left(\bar{c}_{1}-b \tilde{Y}\right)^{2}}{2} \frac{d}{d \tilde{Y}} \frac{\tilde{Y}}{T^{2}}\right]
$$

to lowest approximation in $\sigma$ when $\tilde{Y}=0(1)$. It follows that

$$
\tilde{Y} \frac{d r_{2}^{(2)}}{d \tilde{Y}}=\frac{\left(\bar{c}_{1}-b \tilde{Y}\right) A^{+2}}{\sigma \bar{c}_{1}^{3} T_{c}}\left[1+O\left(\bar{c}_{1}-b \tilde{Y}\right)\right] \quad \text { as } \quad \tilde{Y} \rightarrow \frac{\bar{c}_{1}}{b} \text {. }
$$


It therefore follows from $(2.13),(2.16),(2.26),(5.2)$, and (5.6) that

$$
\Phi_{2}^{(2)}=\frac{\delta}{\sigma^{2}} \frac{A^{\dagger 2}}{4 T_{c} \bar{c}_{1}^{-3-2}}\left[1+O\left(\bar{c}_{1}-b \tilde{Y}\right)\right], \quad \text { as } \quad \tilde{Y} \rightarrow \frac{\bar{c}_{1}}{b},
$$

and similarly from $(2.24),(5.3)$, and (5.5) that

$$
\Psi_{2}^{(2)}=-\frac{\delta}{\sigma^{2}}\left[J_{1}+0\left(b \tilde{Y}-\tilde{c}_{1}\right)\right] \quad \text { as } \quad Y+\frac{\bar{c}_{1}}{b} \text {. }
$$

where $J_{1}$ is an $0(1)$ constant.

6. THE CRITICAL LAYER

Equation (4.16) shows that the edge layer solution becomes singular in the critical layer where $\tilde{Y}=b / \bar{c}_{1}$. The governing equations therefore have to be rescaled to obtain a bounded result in this region. The thickness of the linear small-growth-rate critical layer is of the order of that growth rate divided by the mean velocity gradient times the real part of the wave number, i.e.,

$$
0\left(\frac{\left(\frac{\sigma^{3}}{\delta}\right)}{(\sigma \delta) \sigma}\right)=0\left(\frac{\sigma}{\delta^{2}}\right) .
$$

It therefore follows from (2.10) and (2.13) that the appropriate transverse coordinate in this region is

$$
\bar{Y} \equiv\left(\tilde{Y}-\frac{\bar{c}_{1}}{b}\right) \frac{\delta}{\sigma} \text {. }
$$

Equations (2.16) to $(2.20),(2.33)$ to $(2.36),(4.1),(4.15),(4.16)$, to $(5.3)$, and $(5.8)$ to $(5.10)$ suggest that the flow in this region should expand like 


$$
\begin{aligned}
& u=1-\sigma(b+\ldots . .) \frac{\bar{c}_{1}}{b}-\frac{\sigma^{2}}{\delta} b \bar{Y}-\left(\frac{\sigma \bar{c}_{1}}{2 \delta}\right)^{2}+\frac{\varepsilon \delta}{\sigma} \frac{\operatorname{ReA}_{e}{ }^{\dagger}{ }^{i \bar{X}}}{T_{c} \bar{\alpha}_{1} \bar{c}_{1}}+\varepsilon \bar{u}_{1} \\
& +\frac{\delta^{3} \varepsilon^{2}}{\sigma^{4}} \bar{u}_{2}+\ldots . \\
& \bar{v}=\frac{\varepsilon \delta}{\sigma} \operatorname{Re}\left[\left(\frac{b \bar{Y}}{\bar{c}_{1}^{2}}-\frac{\bar{\alpha} T_{c}^{2}}{c_{1}}\right) i A^{\dagger}-\frac{1}{\bar{c}_{1}^{2} \bar{\alpha}_{1}} A^{\dagger^{\prime}}\right] e^{i \bar{X}}-\frac{\delta^{3} \varepsilon^{2}}{\sigma^{4}} \frac{1}{2 \bar{\alpha}^{-{ }^{3}{ }_{1} T_{c}}} \operatorname{Re} i A^{\dagger 2} e^{2 i \bar{X}} \\
& +\varepsilon \bar{v}_{1}+\cdot \cdot \cdot, \\
& \theta=T_{c}+\frac{\sigma}{\delta}(\gamma-1) b \tilde{Y}+\frac{\varepsilon \delta}{\sigma^{2}} \bar{\tau}_{1}+. . .,
\end{aligned}
$$

and

$$
p^{T / Y}=1+\varepsilon \operatorname{Re} A^{\dagger} e^{i \bar{X}}+\frac{\varepsilon \sigma}{\delta} \bar{\pi}_{1}+\ldots .,
$$

where the passive terms involving $\ln (\sigma / \delta)$ have been incorporated into $\bar{u}_{1}$, etc., and

$$
\bar{v} \equiv v\left(\frac{\delta}{\sigma}\right)^{2}
$$

Then the critical layer solution will match with the "outer" edge layer solution if we require that

$$
\begin{aligned}
& \bar{\tau}_{1} \rightarrow-\frac{(Y-1)}{T_{c} \bar{\alpha}_{1} \bar{c}_{1}} \operatorname{Re} A^{\dagger} e^{i \bar{X}}, \\
& \frac{\partial \bar{u}_{1}}{\partial \bar{Y}}-\frac{b}{\bar{\alpha}_{1}\left(T_{c} \bar{c}_{1}\right)^{2}} \operatorname{ReA} e^{i \bar{X}},
\end{aligned}
$$

as

$$
\bar{Y} \rightarrow \pm \infty,
$$

and 


$$
\begin{array}{r}
\Delta \bar{u}_{1} \equiv \lim _{\bar{Y} \rightarrow \infty}\left[\bar{u}_{1}(\bar{Y})-\bar{u}_{1}(-\bar{Y})\right]=-\frac{1-\bar{c}_{1}^{-2}(Y-1)^{2}}{T_{c} \bar{c}_{1}} \operatorname{Re}\left(\phi^{+}-\phi^{-}\right) A^{\dagger} e^{i \bar{X}} \\
+ \text { higher harmonics. }
\end{array}
$$

The expansion coefficients $\bar{u}_{1}, \bar{v}_{1}, \bar{\tau}_{1}$, etc. are functions of $X, \bar{Y}$, and $x_{1}$ only. They are determined by the inviscid vorticity, energy and continuity equations, which can be written as

$$
\bar{D}_{\omega}-\frac{\omega}{\gamma p} \bar{D} p=\frac{\delta^{2} \tilde{Y}}{\theta_{Y p}}\left[\left(\bar{\alpha} \theta_{\bar{X}}+\frac{\sigma^{2}}{\delta} \theta_{x_{1}}\right) p_{\bar{Y}}-\theta_{\bar{Y}}\left(\bar{\alpha} p_{\bar{X}}+\frac{\sigma^{2}}{\delta} p_{x_{1}}\right)\right] \text {, }
$$

and

$$
\frac{1}{\gamma p} \bar{D} p=\frac{1}{(\gamma-1) \theta} \overline{D \theta}=-\left(\bar{\alpha} u_{\bar{X}}-\frac{\tilde{Y}}{\bar{T}} \bar{v}_{\bar{Y}}-\frac{\sigma^{2}}{\delta} u_{x_{I}}\right) \text {, }
$$

where we have put

$$
\bar{D} \equiv \bar{\alpha}(u-c) \frac{\partial}{\partial \bar{X}}-\bar{v} \frac{\tilde{Y}}{T} \frac{\partial}{\partial \bar{Y}}+\frac{\sigma^{2}}{\delta} u \frac{\partial}{\partial x_{1}},
$$

and

$$
\omega=\frac{\delta^{2}}{\sigma} \frac{\tilde{Y}}{\bar{T}} \frac{\partial u}{\partial \bar{Y}}+\frac{\sigma^{3}}{\delta^{2}}\left(\bar{\alpha} \frac{\partial \bar{v}}{\partial \bar{X}}+\frac{\sigma^{2}}{\delta} \bar{v}_{x_{1}}\right)
$$

is the vorticity.

The crucial step in the analysis is to choose the relation between the amplitude scale $\varepsilon$ and the wavelength scale $\sigma$ so that the noninear terms produce a critical layer velocity jump of the same order as the velocity jump due to linear effects, i.e., $O(\varepsilon)$. A little experimentation shows that nonlinear effects will influence the $O(\varepsilon)$ term in $u$ (both through the cross stream derivative in $\bar{D}$ and the vorticity source term on the right side of $(6.10))$ if we take 


$$
\varepsilon=\frac{\sigma^{3}}{\delta^{2}}
$$

Then $\bar{u}_{1}$ and $\bar{\tau}_{1}$ will satisfy

$$
\bar{D}\left(\frac{\partial \bar{u}_{1}}{\partial \bar{Y}}-\frac{b^{2} \bar{Y}}{T_{c} \bar{c}_{1}}\right)=-\bar{\alpha}\left[\frac{\partial \bar{\tau}_{1}}{\partial \bar{Y}}+b(\gamma-1)\right] \operatorname{Re} i A^{\dagger} e^{i \bar{X}}
$$

and

$$
\overline{\mathscr{D}}\left(\bar{\tau}_{1}+b(\gamma-1) \bar{Y}\right)=0
$$

where we have put

$$
\begin{aligned}
\bar{D} \equiv \frac{\partial}{\partial x_{1}}-\bar{\alpha}\left(b \bar{Y}-\frac{1}{T_{c} \bar{\alpha} \bar{c}_{1}} \operatorname{Re} A^{\dagger} e^{i \bar{X}}\right) \frac{\partial}{\partial \bar{X}} \\
-\frac{1}{T_{c}}\left\{\operatorname{Re}\left[\left(\frac{\bar{Y}}{\bar{c}_{1}}-\frac{\bar{\alpha} T_{c}^{2}}{b}-\frac{1}{b T_{c} \bar{\alpha}^{-2} \bar{c}_{1}} \operatorname{Re} A^{\dagger} e^{i \bar{X}}\right) i A^{\dagger}-\frac{1}{\bar{c}_{1} b \bar{\alpha}_{1}} A^{{ }^{\prime}}\right] e^{i \bar{X}}\right\} \frac{\partial}{\partial \bar{Y}}
\end{aligned}
$$

This can be greatly simplified and put into a more standard form by taking $x_{1}$, $\bar{x}$, and

$$
\bar{Y}_{0} \equiv-\bar{Y}+\frac{1}{b T_{c} \bar{\alpha}_{1} \bar{c}_{1}} \operatorname{ReA} e^{i \bar{X}}
$$

as new independent variables, in which case $\overline{\mathscr{D}}$ becomes

$$
\overline{\mathscr{D}}=\frac{\partial}{\partial x_{1}}+\bar{\alpha} b \bar{Y}_{0} \frac{\partial}{\partial \bar{X}}-\frac{T_{c} \bar{\alpha}_{1}}{b}\left(\operatorname{Re} i A^{\dagger} e^{i \bar{X}}\right) \frac{\partial}{\partial \bar{Y}_{0}} .
$$

It now follows from (3.5), (4.13), (4.14), and (6.9) that

$$
\frac{1}{\pi} \int_{0}^{2 \pi} \int_{-\infty}^{\infty} e^{-i \bar{X}} \frac{\partial \bar{u}_{1}}{\partial \bar{Y}_{0}} d \bar{Y}_{0} d \bar{X}=-\frac{1}{\bar{c}_{1} \bar{\alpha}_{1} \Gamma} A^{\dagger^{\prime}} .
$$


where we have put

$$
\frac{1}{\mathrm{r}} \equiv-\frac{1}{T_{c} \bar{c}_{1}} \bar{\alpha}_{1}\left(2-\bar{c}_{1}^{3} \int_{0}^{\infty} q d n\right)
$$

To put these results in a more universal form, we introduce the new variables

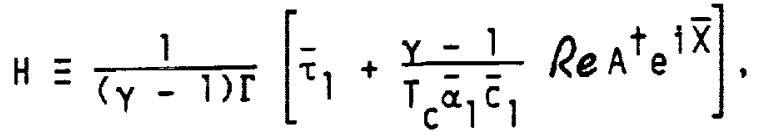

$$
\begin{aligned}
& \Omega \equiv-\frac{T_{c} \bar{c}_{1}}{b \Gamma}\left[\frac{\partial \bar{u}_{1}}{\partial \bar{y}_{0}}+\frac{b}{\bar{\alpha}_{1}\left(T_{c} \bar{c}_{1}\right)^{2}} \operatorname{Re} A^{\dagger} e^{i \bar{x}}\right] \text {, } \\
& A \equiv \frac{T_{c} A^{\dagger} e^{i x_{0}}}{\Gamma^{2}} \text {. } \\
& Y \equiv \frac{b \bar{Y}_{0}}{\Gamma}, \\
& x \equiv \bar{x}-x_{0} \text {, }
\end{aligned}
$$

and

$$
\bar{x} \equiv \Gamma \bar{\alpha}_{1} x_{1}-x_{0} .
$$

Then $H$ and $\Omega$ satisfy the homogeneous boundary conditions

$$
H, \Omega \rightarrow 0 \text { as } Y \rightarrow \pm \infty,
$$

and $(6.15),(6.16),(6.19)$, and $(6.20)$ become

$$
\begin{gathered}
\mathscr{D} \Omega=\left(1-r+r \frac{\partial H}{\partial Y}\right) \operatorname{Re} i A e^{i X}, \\
\mathscr{D H}=-\operatorname{Re} i A e^{i X},
\end{gathered}
$$

and

$$
\frac{1}{\pi} \int_{-\infty}^{\infty} \int_{0}^{2 \pi} \Omega e^{-i X} d y d X=1 \frac{d A}{d \bar{x}}
$$


where we have put

$$
\mathscr{D} \equiv \frac{\partial}{\partial \bar{x}}+Y \frac{\partial}{\partial X}-\left(\operatorname{Re}_{i A e^{i X}}\right) \frac{\partial}{\partial Y}
$$

and

$$
r \equiv(\gamma-1) \bar{c}_{1}=T_{c}-1 \text {. }
$$

It is easy to see from these equations that

$$
H \rightarrow-\operatorname{Re} \frac{A e^{i X}}{Y-i \vec{k}},
$$

and

$$
\Omega \rightarrow(1-r) \operatorname{Re} \frac{A e^{i X}}{Y-i \bar{\kappa}}
$$

when

$$
A \rightarrow e^{\bar{k} \bar{x}} \quad \text { as } \quad \bar{x} \rightarrow-\infty,
$$

where

$$
\bar{\kappa} \equiv(1-r) \pi
$$

is the scaled (and normalized) linear growth rate. The solution to these equations can therefore be made to satisfy the upstream matching condition (2.48) If we choose the, as yet, unspecified real constants $x_{0}$ and $x_{0}$ to be

$$
x_{0}=-\arg a \text {, }
$$

and

$$
x_{0}-\frac{1}{k} \ln \frac{\Gamma^{2}}{|a|} .
$$

7. NUMERICAL COMPUTATIONS

The coupled nonlinear evolution equations (6.29) and (6.30) must be solved numerically. It is easy to see from these equations and the upstream boundary condition (6.36) that the solution $A$ will remain real for all values of $\bar{x}$. Then since $\Omega$ and $H$ are periodic in $X$, we expand them in Fourier series 


$$
\begin{aligned}
& \Omega=\frac{1}{2} \sum_{h=-\infty}^{\infty} \Omega_{n} e^{i n x} \\
& H=\frac{1}{2} \sum_{h=-\infty}^{\infty} H_{n} e^{i n X}
\end{aligned}
$$

with $\Omega_{-n}=\Omega_{n}^{*}, H_{-n}=-H_{n}^{*}$ (where the asterisk denotes the complex conjugate) to obtain

$$
\begin{aligned}
\left(\frac{\partial}{\partial \bar{x}}+i n Y\right) \Omega_{n}+\frac{1}{2} A \frac{\partial}{\partial Y}\left[\Omega_{n+1}+r H_{n+1}-\Omega_{n-1}-r H_{n-1}\right] & =i \delta_{n, 1}(1-r) A, \\
\left(\frac{\partial}{\partial \bar{x}}+i n Y\right) H_{n}+\frac{1}{2} A \frac{\partial}{\partial Y}\left(H_{n+1}-H_{n-1}\right) & =-i \delta_{n, 1} A,
\end{aligned}
$$

where $A$ satisfies $(6.36)$,

$$
\begin{gathered}
H_{n}-\frac{\delta_{n, 1^{A}}}{Y-i \bar{k}}, \\
\Omega_{n}-\frac{(1-r) \delta_{n, 1^{A}}}{Y-i \bar{k}},
\end{gathered}
$$

as $\bar{x} \rightarrow-\infty$, and

$$
\eta_{m} \int_{-\infty}^{\infty} \Omega_{1} d Y=\frac{d A}{d \bar{x}}
$$

We solved (7.3) to (7.7) numerically using a procedure similar to the one used by Hanes (1985) and Goldstein, Durbin, and Leib (1987). Rather than mapping the Infinite domain $-\infty<Y<\infty$ into a finite domain, they simply solved the equations over a finite range, say $-N \leq Y \leq N$, and used the asymptotic behavior of the solutions at $Y= \pm \infty$ to obtain an accurate approximation to the cross stream integral ((7.7) in the present case).

By using (6.29) and (6.30) to generate asymptotic expansions it is easy to show that

$$
H_{0}, \Omega_{0}=O\left(Y^{-3}\right)
$$




$$
\begin{gathered}
\Omega_{1}-\frac{(1-r)}{Y} A+\frac{j(1-r)}{Y^{2}} \frac{d A}{d \bar{x}}+O\left(Y^{-3}\right) \\
H_{1}--\frac{1}{Y} A-\frac{1}{Y^{2}} \frac{d A}{d \bar{x}}+O\left(Y^{-3}\right) \\
\Omega_{n}, H_{n}=O\left(\frac{1}{Y^{n+1}}\right) \text { for } n \geq 2
\end{gathered}
$$

as $Y- \pm \infty$.

It follows that (7.7) can be approximated by

$$
\left[1-\frac{2}{N}(1-r)\right] \frac{d A}{d \bar{x}}=\ln \int_{-N}^{N} Q_{1} d y+O\left(N^{-3}\right)
$$

The numerical technique used to solve (7.3), (7.4), and (7.12) was similar to that used by Goldstein and Hultgren (1988). Equations (7.3) and (7.4) were solved for $0 \leq n \leq \bar{n}$ where $\bar{n}$ was chosen so that the maximum absolute values of $H_{\bar{n}}$ and $\Omega_{\bar{n}}$ remained below a certain present tolerance. The $Y$ derivatives in (7.3) and (7.4) were discretized using second order central difference approximations and Simpson's one-third rule was used for the integrals in (7.12). The calculation was started in the upstream linear region where $A, \Omega_{n}$, and $H_{n}$ are accurately approximated by (7.5) and (7.6) and marched forward in $\bar{x}$ through a predictor corrector procedure. A third order scheme was used for (7.12). The predictor step for (7.3) and (7.4) consisted of an Adams-Moulton-Bashforth second order explicit approximation for the nonlinear and inhomogeneous terms and the Crank-Nicholson (second order implicit) approximation for the mean flow convection terms. The corrector step was fully of the Crank-Nicholson type. The combined corrector steps for (7.3), (7.4), and (7.12) were then iterated until the solution at the next streamwise station was obtained to within a preset tolerance. 


\section{NUMERICAL RESULTS AND DISCUSSION}

Goldstein, Durbin, and Leib (1987) considered the nonlinear evolution of a two-dimensional instability wave in a weak adverse pressure gradient boundary layer. Equation (6.29) reduces to their result in the limit where $r \rightarrow 0$ and equations (6.29) and (6.30) become decoupled. They found that nonlinear effects always reduce the growth rate of the linear instability wave, driving it toward an equilibrium state.

The scaled instability wave amplitude is plotted as a function of the scaled and normalized streamwise coordinate $\bar{x}$ for vartous values of $r$ in figure 1. The corresponding instability wave growth rates, $A \bar{x} / A$, are shown in figure 2. As in Goldstein et al. (1987) the growth rates initially follow the linear growth until the amplitude becomes large enough for nonlinear effects to come into play, but now the nonlinear effects can cause the growth rate to become larger than the linear growth presumably because compressibility effects, i.e., the Bjerknes forces, act as a vorticity source within the critical layer. Notice that the growth augmentation increases with increasing $r$ and only occurs when $r$ exceeds a certain finite value, say ro.

Figure 3 shows the rollup of the vorticity contours in the $X-Y$ plane at various (increasing) values of $\bar{x}$. The rollup is not too different from that found by Goldstein et al. with the principal difference being the formation of an additional counter rotating vortex core. The results are replotted in figure 4 versus the more physical coordinates $X$ and $-\bar{Y} b / \Gamma$ for

$$
\frac{\Gamma}{T_{c \bar{\alpha}_{1} \bar{c}_{1}}}=\frac{1}{2} \text {. }
$$

Notice that they now look considerably different. 


\section{REFERENCES}

Cowley, S. and Hall, P. (1988) On the Instability of Hypersonic Flow Past a Wedge. ICASE report no. 88-72.

Cowley, S. and Hall, P. (1989) On the Instability of Hypersonic Flow Past a Wedge. 3rd IUTAM Symposium on Laminar-Turbulent Transition, Toulouse, France.

Goldstein, M.E. (1984) Aeroacoustics of Turbulent Shear Flows. Ann. Rev. Fluid Mech., vol. 16, pp. 263-285.

Goldstein, M.E., Durbin, P.A., and Leib, S.J. (1987) Rollup of Vorticity in Adverse-Pressure-Gradient Boundary Layers. J. Fluld Mech., vol. 183, pp. $325-342$.

Goldstein, M.E. and Hultgren, L.S. (1988) Nonlinear Spatial Evolution of an Exceptionally Excited Instability Wave in a Free Shear Layer. J. Fluid Mech., vol. 197, pp. 295-330.

Goldstein, M.E. and Leib, S.J. (1989) Nonlinear Evolution of Oblique Waves on Compressible Shear Layers. J. Fluid Mech., vol. 207, pp. 73-96.

Schlichting, H. (1960) Boundary Layer Theory, McGraw Hill.

Hanes, P.H. (1985) Nonlinear Instability of a Ramsby-Wave Critical Layer. J. Fluid Mech., vol. 161, pp. 493-511. 
Mack, L.M. (1984) Boundary-Layer Linear Stability Theory. In Special Course on Stability and Transition of Laminar Flow. AGARD report 709.

Mack, L.M. (1987) Review of Linear Compressible Stability Theory. In Stability of Time Dependent and Spatially Varying Flows. Eds. D.L. Dwoyer and M.Y. Hussaini, Springer-Verlag.

Stewartson, K. (1964) Theory of Laminar Boundary Layers in Compressible Fluids. Oxford University Press.

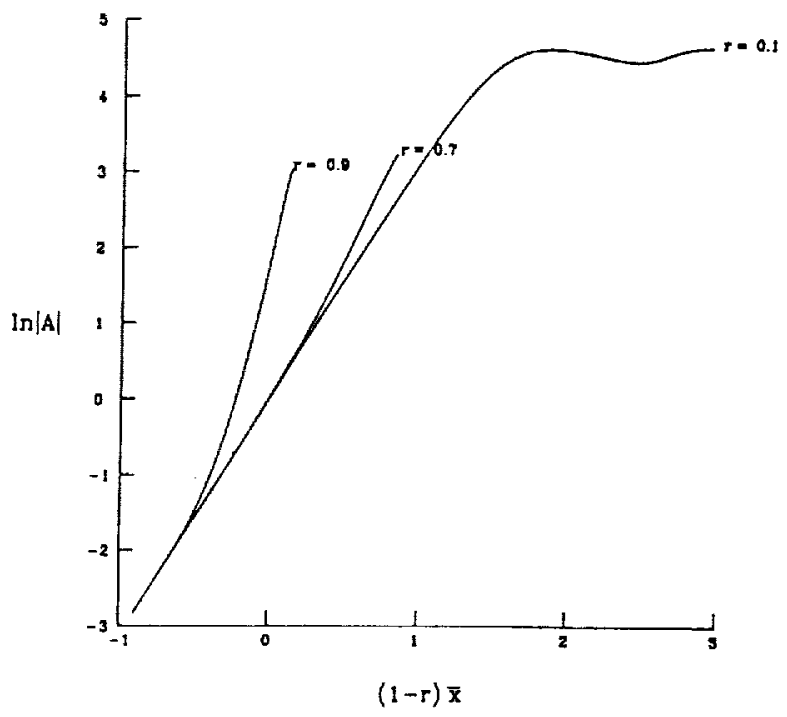

FIGURE $1 .-\ln |A|$ VERSUS $(1-r) \bar{x}$ FOR $r=0.1,0.7$, AND 0.9.

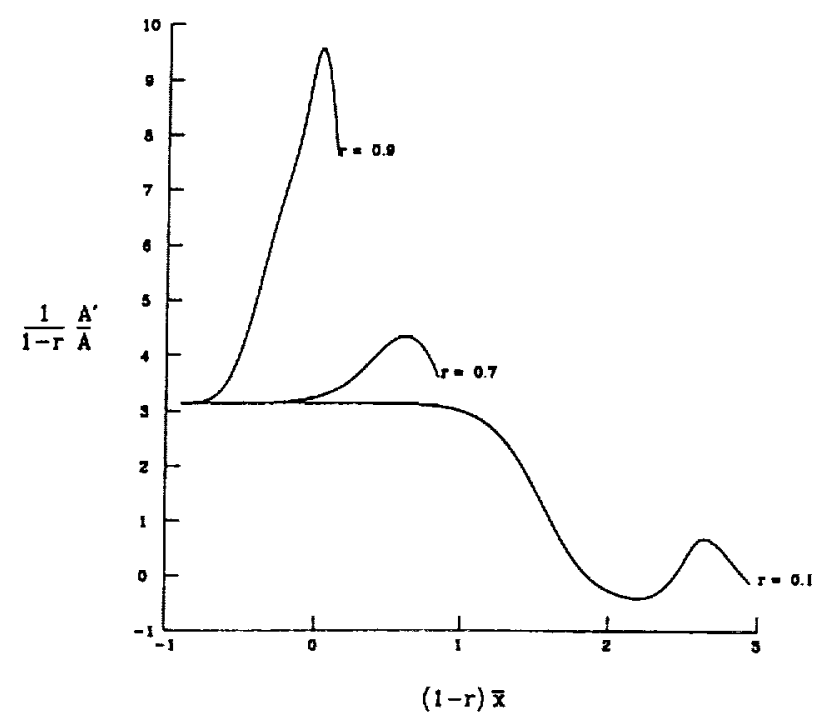

FIGURE 2. $-A^{\prime} /(1-r) A$ VERSUS $(1-r) \bar{x}$ FOR $r=0.1$. 0.7 . AND 0.9. 

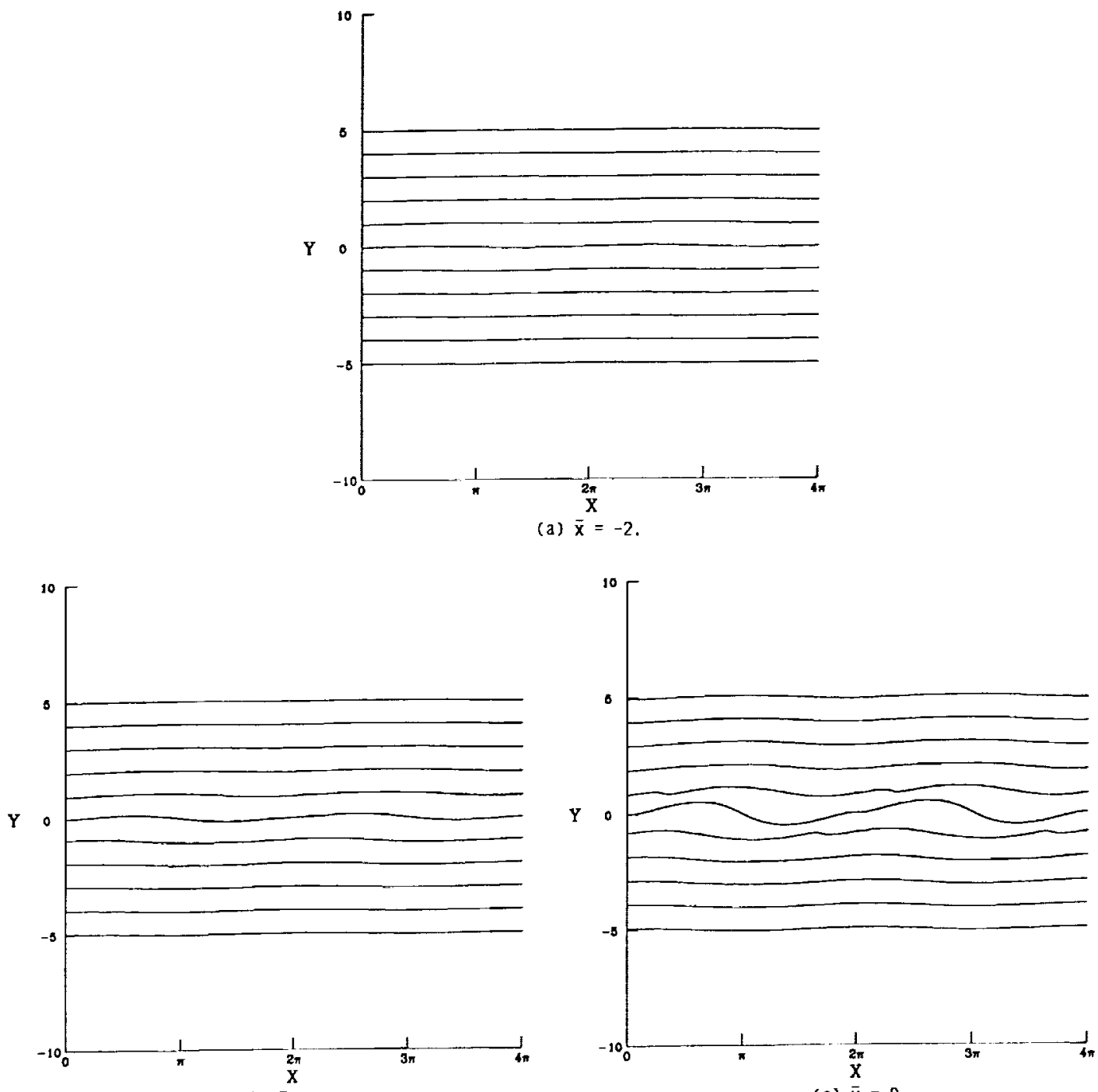

(b) $\bar{x}=-1$

(c) $\bar{x}=0$.
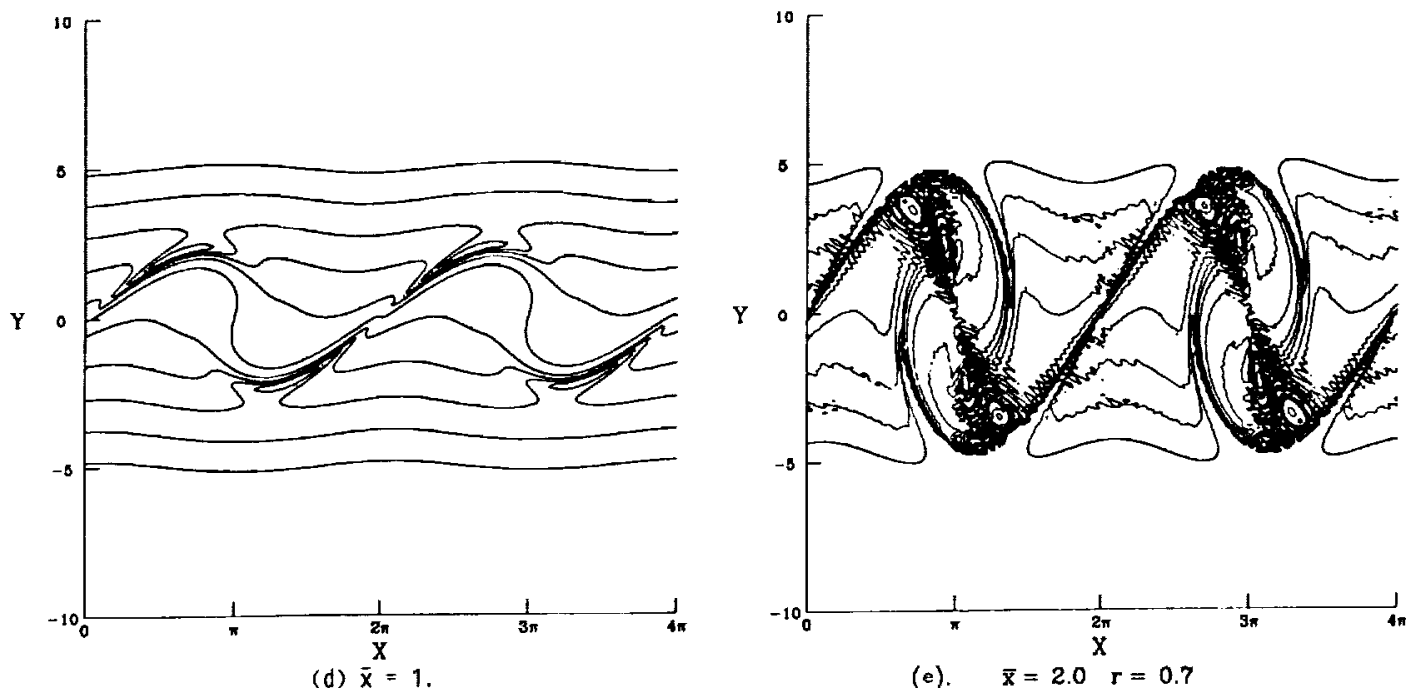

(e). $\bar{x}=2.0^{\circ} \quad r=0.7$

FIGURE 3, - VORTICITY CONTOURS IN THE $(X, Y)$-PLAME FOR $r=0.7$ AND CONTOUR VALUES $-10,-9, \ldots 9$ AND 10. 


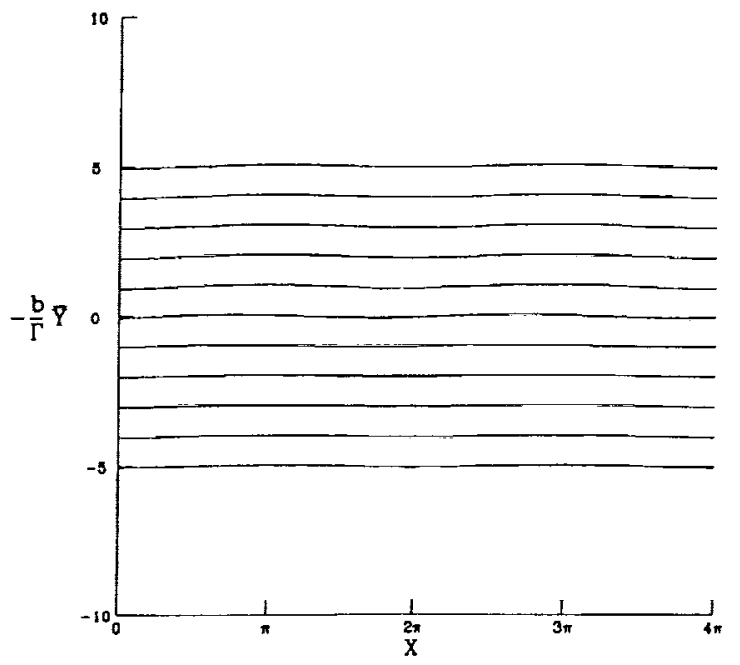

(a) $\bar{x}=-2$.

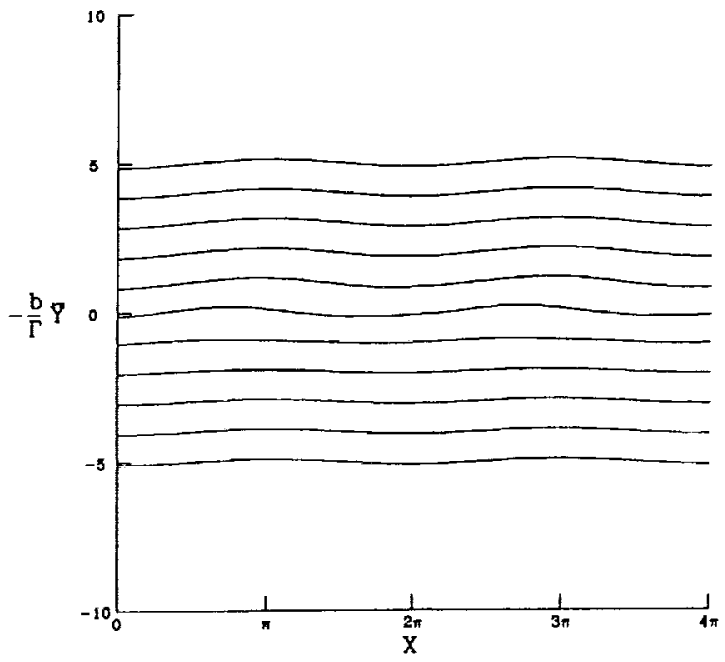

(b) $\bar{x}=-1$.

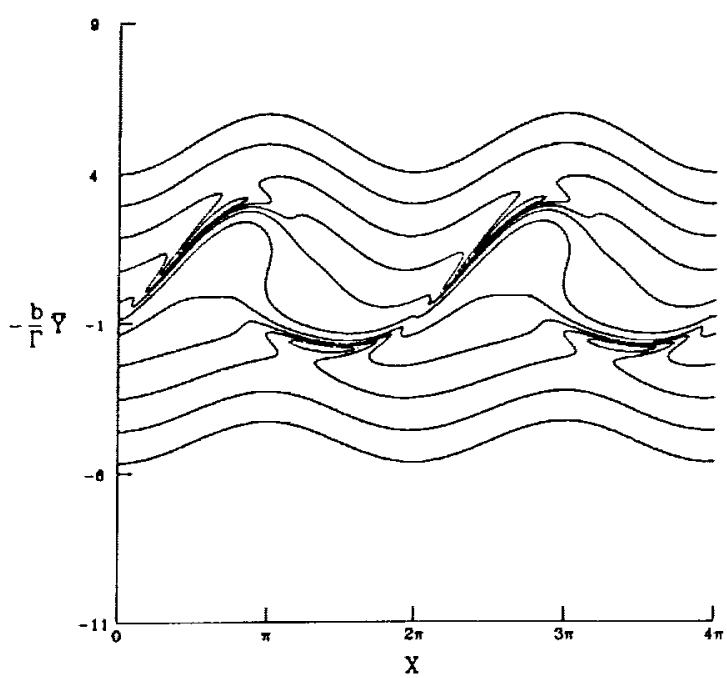

(d) $\bar{x}=1$.

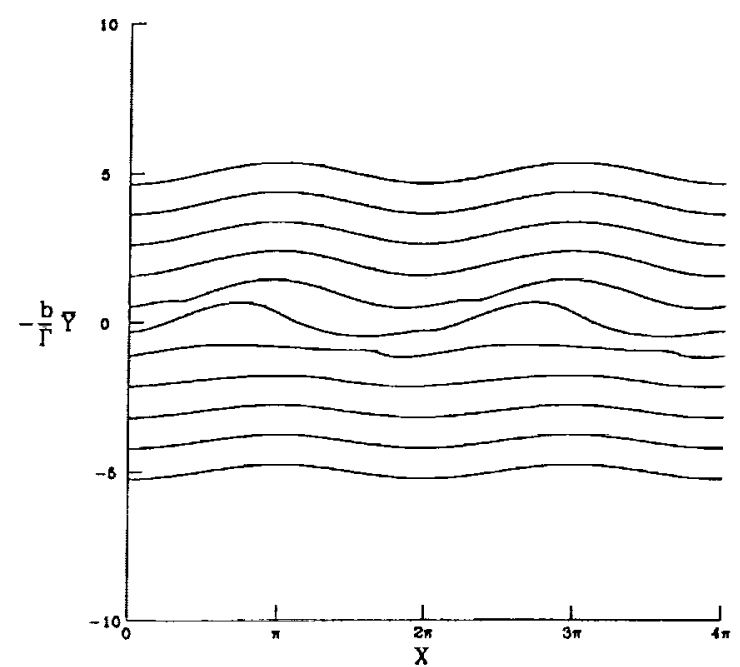

(c) $\bar{x}=0, \quad 7$

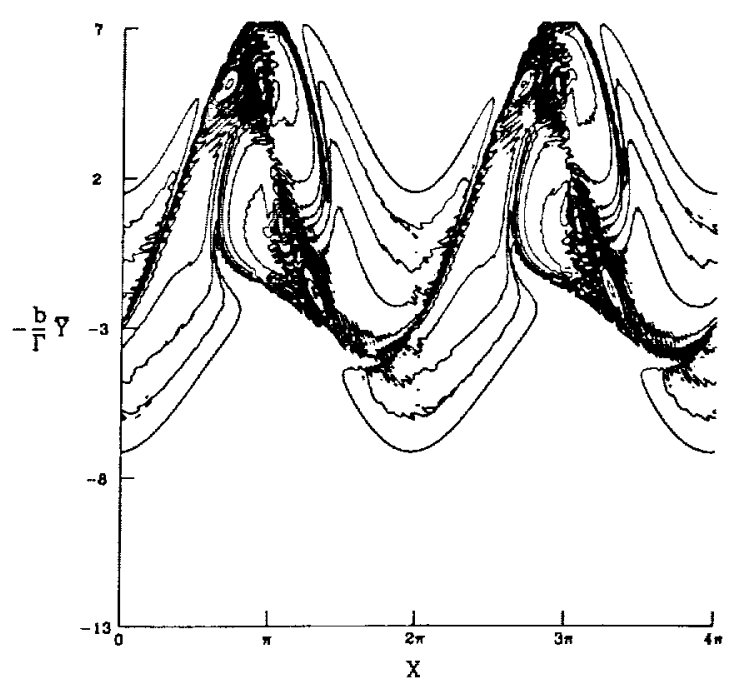

(e). $\quad \bar{x}=2.0 \quad \mathrm{r}=0.7$

FIGURE 4. - VORTICITY CONTOURS IN THE $(X,-$ Y/D)-PLANE FOR $r=0.7$ AND CONTOUR VALUES $-10,-9, \ldots 9$ AND 10. 


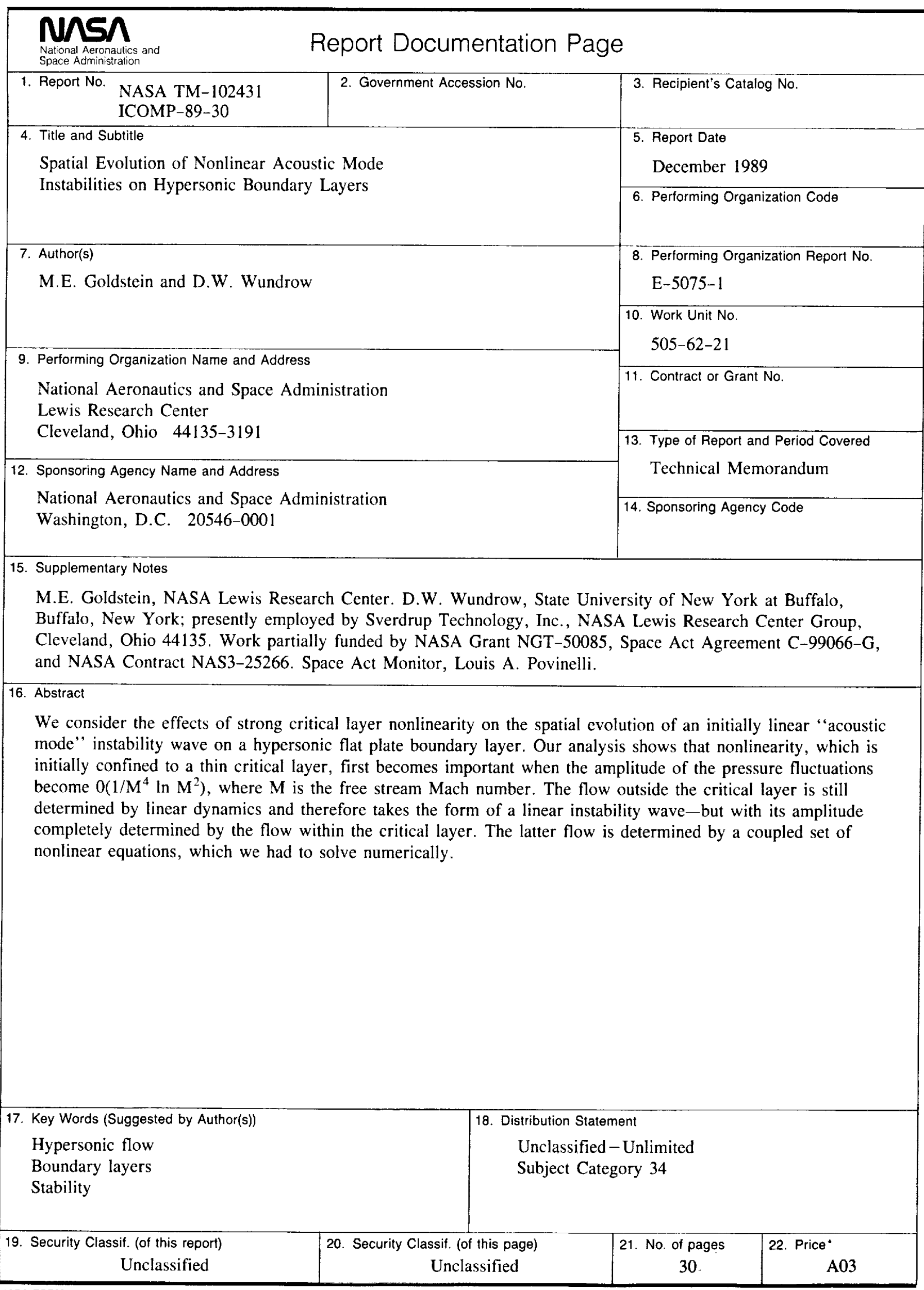




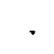

. 